\title{
Things We Do For No Reason: Electrolyte Testing in Pediatric Acute Gastroenteritis
}

\author{
Carrie H. Lind, MD*, David P. Johnson, MD
}

Division of Pediatric Hospital Medicine, Monroe Carell Jr. Children's Hospital at Vanderbilt, Vanderbilt University, Nashville, Tennessee.

The "Things We Do for No Reason" (TWDFNR) series reviews practices which have become common parts of hospital care but which may provide little value to our patients. Practices reviewed in the TWDFNR series do not represent "black and white" conclusions or clinical practice standards, but are meant as a starting place for research and active discussions among hospitalists and patients. We invite you to be part of that discussion.

Acute gastroenteritis (AGE) remains a substantial cause of childhood illness and is 1 of the top 10 reasons for pediatric hospitalization nationwide. In the United States, AGE is responsible for $10 \%$ of hospital admissions and approximately 300 deaths annually. ${ }^{1}$ The American Academy of Pediatrics (AAP) and other organizations have emphasized supportive care in the management of AGE. Routine diagnostic testing has been discouraged in national guidelines except in cases of severe dehydration or an otherwise complicated course. Despite AGE guidelines, diagnostic laboratory tests are still widely used even though they have been shown to be poor predictors of dehydration. Studies have shown that high test utilization in various pediatric disease processes often influences the decision for hospitalization without improvement in patient outcome. In children with AGE, the initial and follow-up laboratory tests may not only be something that we do for no reason, but something that is associated with more risk than benefit.

An 18-month-old healthy male is brought to the emergency department (ED) with a chief complaint of 2 days of nonbloody, nonbilious emesis and watery diarrhea. He has decreased energy but smiles and plays for a few minutes. He has had decreased wet diapers. His exam is notable for mild tachycardia, mildly dry lips, and capillary refill of 3 seconds. A serum electrolyte panel is normal except for a sodium of $134 \mathrm{mEq} / \mathrm{L}$, a bicarbonate of $16 \mathrm{mEq} / \mathrm{L}$, and an anion gap of 18 , which are flagged as abnormal by the electronic medical record. These results prompt intravenous (IV) access, a normal saline bolus, and admission on maintenance fluids overnight. The next morning, his electrolyte panel is repeated, and his sodium is $140 \mathrm{mEq} / \mathrm{L}$ and bicarbonate is $15 \mathrm{mEq} / \mathrm{L}$. He is now drinking well with no further episodes of emesis, so he is discharged home.

*Address for correspondence and reprint requests: Carrie H. Lind, MD, 2200 Children's Way, Room 11208B DOT, Nashville, TN 37232-9000; Telephone: 615936-2119; Fax: (615)875-4623; E-mail: carrie.b.holloway@vanderbilt.edu

Received: March 10, 2017; Revised: August 1, 2017;

Accepted: August 13, 2017; Published online first November 22, 2017

Published online first November 22, 2017

2018 Society of Hospital Medicine DOI 10.12788/jhm.2884

\section{WHY PHYSICIANS MIGHT THINK ELECTROLYTE TESTING IS HELPFUL}

Many physicians across the United States continue to order electrolytes in AGE as a way to avoid missing severe dehydration, severe electrolyte abnormalities, or rare diagnoses, such as adrenal insufficiency or new-onset diabetes, in a child. Previous studies have revealed that bicarbonate and blood urea nitrogen (BUN) may be helpful predictors of severe dehydration. A retrospective study of 168 patients by Yilmaz et al. ${ }^{2}$ showed that BUN and bicarbonate strongly correlated with dehydration severity $(P<.00001$ and $P=.01$, respectively). $A$ 97-patient prospective study by Vega and Avner ${ }^{3}$ showed that bicarbonate $<17$ can help in predicting percent body weight loss (PBWL) (sensitivity of 77\% for PBWL 6-10 and $94 \%$ for PBWL >10).

In AGE, obtaining laboratory data is often considered to be the more conservative approach. Some attribute this to the medical education and legal system rewarding the uncovering of rare diagnoses, ${ }^{4}$ while others believe physicians obtain laboratory data to avoid missing severe electrolyte disorders. One author notes, "physicians who are anxious about a patient's problem may be tempted to do something - anything-decisive in order to diminish their own anxiety. ${ }^{\prime 5}$ Severe electrolyte derangements are common in developing countries ${ }^{6}$ but less so in the United States. A prospective pediatric dehydration study over 1 year in the United States demonstrated rates of $6 \%$ and $3 \%$ of hypo- and hypernatremia, respectively $(n=182)$. Only 1 patient had a sodium level $>160$, and this patient had an underlying genetic syndrome, and none had hyponatremia $<130$. Hypoglycemia was the most common electrolyte abnormality, which was present in $9.8 \%$ of patients. Electrolyte results changed management in $10.4 \%$ of patients.

\section{WHY ELECTROLYTE TESTING IS GENERALLY NOT HELPFUL}

In AGE with or without dehydration, guidelines from the AAP and other international organizations emphasize supportive care in the management of AGE and discourage routine diagnostic testing..$^{8-10}$ Yet, there continues to be wide variation in AGE management. ${ }^{11-13}$ Most AGE cases presenting to an outpatient setting or ED are uncomplicated: age $>6$ months, nontoxic appearance, no comorbidities, no hematochezia, diarrhea $<7$ days, and mild-to-moderate dehydration.

Steiner et al. ${ }^{14}$ performed a systematic meta-analysis of the precision and accuracy of symptoms, signs, and laboratory tests for evaluating dehydration in children. They concluded that a standardized clinical assessment based on physical exam (PE) 
TABLE. Clinical Dehydration Scale for Children (Total Score From 0-8) ${ }^{26}$

\begin{tabular}{lccc}
\hline Characteristics & $\mathbf{0}$ & $\mathbf{1}$ & $\mathbf{2}$ \\
\hline General appearance & Normal & $\begin{array}{c}\text { Thirsty, restless, or lethargic, } \\
\text { but irritable when touched }\end{array}$ & $\begin{array}{c}\text { Drowsy, limp, cold, or sweaty, } \\
\text { and/or comatose }\end{array}$ \\
\hline Eyes & Normal & Slightly sunken & Very sunken \\
\hline Mucous membranes & Moist & Sticky & Dry \\
\hline Tears & Tears & Decreased tears & Absent
\end{tabular}

NOTE: A score of 0 represents no dehydration; a score of 1-4, some dehydration; and a score of 5-8, moderate or severe dehydration.

findings more accurately classifies the degree of dehydration than laboratory testing. Steiner et al ${ }^{14}$ specifically analyzed the works by Yilmaz et al. ${ }^{2}$ and Vega and Avner, ${ }^{3}$ and determined that the positive likelihood ratios for $>5 \%$ dehydration resulting from a BUN $>45$ or bicarbonate $<17$ were too small or had confidence intervals that were too wide to be clinically helpful alone. Therefore, Steiner et al. ${ }^{14}$ recommended that laboratory testing should not be considered definitive for dehydration.

Vega and Avner ${ }^{3}$ found that electrolyte testing is less helpful in distinguishing between $<5 \%$ (mild) and $5 \%$ to $10 \%$ (moderate) dehydration compared to PBWL. Because both mild and moderate dehydration respond equally well to oral rehydration therapy (ORT), electrolyte testing is not helpful in managing these categories. Many studies have excluded children with hypernatremia, but generally, severe hypernatremia is uncommon in healthy patients with AGE. In most cases of mild hypernatremia, ORT is the preferred resuscitation method and is possibly safer than IV rehydration because ORT may induce less rapid shifts in intracellular water. ${ }^{15}$

Tieder et al..$^{16}$ demonstrated that better hospital adherence to national recommendations to avoid diagnostic testing in children with AGE resulted in lower charges and equivalent outcomes. In this large, multicenter study among 27 children's hospitals in the Pediatric Hospital Information System (PHIS) database, only $70 \%$ of the 188,000 patients received guideline-adherent care. Nonrecommended laboratory testing was common, especially in the admitted population. Electrolytes were measured in $22.1 \%$ of the ED and observation patients compared with $85 \%$ of admitted patients. Hospitals that were most guideline adherent in the ED demonstrated $50 \%$ lower charges. The authors estimate that standardizing AGE care and eliminating nonrecommended laboratory testing would decrease admissions by $45 \%$ and save more than $\$ 1$ billion per year in direct medical costs. ${ }^{16}$ In a similar PHIS study, laboratory testing was strongly correlated with the percentage of children hospitalized for AGE at each hospital $(r=0.73, P<.001)$. Results were unchanged when excluding children $<1$ year of age $(r=0.75, P<.001)$. In contrast, the mean testing count was not correlated with return visits within 3 days for children discharged from the ED $(r=0.21, P=.235)$, nor was it correlated with hospital length of stay $(r=-0.04, P=.804)$ or return visits within 7 days $(r=0.03, P=.862)$ for hospitalized children..$^{12} \mathrm{In}$ addition, Freedman et al..$^{17}$ revealed that the clinical dehydration score is independently associated with successful ED discharge without revisits, and laboratory testing does not prevent missed cases of severe dehydration.

Nonrecommended and often unnecessary laboratory testing in AGE results in IV procedures that are sometimes repeated because of abnormal values. "Shotgun testing," or ordering a panel of labs, can result in abnormal laboratory values in healthy patients. Deyo et al. ${ }^{18}$ cite that for a panel of 12 laboratory values, there is a $46 \%$ chance of having at least 1 abnormal lab, even in healthy patients. These false-positive results can then drive further testing. In AGE, an abnormal bicarbonate may drive repeat testing to confirm normalization, but the bicarbonate may actually decrease once IV fluid therapy is initiated due to excessive chloride in isotonic fluids. Coon et al. ${ }^{19}$ have shown that seemingly innocuous testing or screening can lead to overdiagnosis, which can cause physical and psychological harm to the patient and has financial implications for the family and healthcare system. While this has not been directly investigated in pediatric AGE, it has been studied in common pediatric illnesses, including pneumonia and urinary tract infections. ${ }^{20,21}$ For children, venipuncture and IV placements are often the most distressful components of a hospital visit and can affect future healthcare encounters, making children anxious and distrustful of the healthcare system. ${ }^{22,23}$

\section{WHY ELECTROLYTE TESTING MIGHT BE HELPFUL}

Electrolyte panels may be useful in assessing children with severe dehydration (scores of 5-8 on the Clinical Dehydration Scale (CDS) or more than $10 \%$ weight loss) or in complicated cases of AGE (those that do not meet the criteria of age $>6$ months, nontoxic appearance, no comorbidities, no hematochezia, and diarrhea $<7$ days) to guide IV fluid management and correct markedly abnormal electrolytes. ${ }^{14}$

Electrolyte panels may also rarely uncover disease processes, such as new-onset diabetes, hemolytic uremic syndrome, adrenal insufficiency, or inborn errors of metabolism, allowing for early diagnosis and preventing adverse outcomes. Suspicion to investigate such entities should arise during a thorough history and PE instead of routinely screening all children with symptoms of AGE. One should also have a higher level of concern for other disease processes when clinical recovery does not occur within the expected amount of time; symptoms usually resolve within 2 to 3 days but sometimes will last up to a week.

\section{WHAT WE SHOULD DO INSTEAD}

A thorough history and PE can mitigate the need for electrolyte testing in patients with uncomplicated AGE. ${ }^{14}$ ORT with repeated clinical assessments, including $P E$, can assist in monitoring clinical improvement and, in rare cases, identify alternative causes of vomiting and diarrhea. ${ }^{24}$ We have included 1 validated and simple-to-use CDS (sensitivity of 0.85 [95\% confidence interval, 0.73-0.97] for an abnormal score; Table). ${ }^{25,26} \mathrm{~A}$ 
standardized use of a CDS, obtained with vital signs, from patient presentation through discharge can help determine initial dehydration status and clinical progression. If typical clinical improvement does not take place, it may be time to evaluate for rarer causes of vomiting and diarrhea. Once a patient is clinically rehydrated or if a patient is tolerating oral fluids so that rehydration is expected, the patient should be ready for discharge, and no further laboratory testing should be necessary.

\section{RECOMMENDATIONS}

- Perform a thorough history and PE to diagnose AGE. ${ }^{8}$

- Clinical assessment of dehydration should be performed upon initial presentation and repeatedly with vital signs throughout the stay using a validated CDS to classify the patient's initial dehydration severity and monitor improvement. Obtain a current patient weight and compare with previously recorded weights, if available. ${ }^{25,26}$

- Laboratory testing in patients with AGE should not be performed unless a patient is classified as severely dehydrated, is toxic appearing, has a comorbidity that increases the likelihood of complications, or is not improving as expected.

- Rehydration via ORT is preferred to an IV in mild and moderate dehydration. ${ }^{15}$

\section{References}

1. Elliott EJ. Acute gastroenteritis in children. BMJ. 2007;334(7583):35-40

2. Yilmaz K, Karabocuoglu M, Citak A, Uzel N. Evaluation of laboratory tests in dehydrated children with acute gastroenteritis. J Paediatr Child Health. 2002;38(3):226-228

3. Vega RM, Avner JR. A prospective study of the usefulness of clinical and laboratory parameters for predicting percentage of dehydration in children. Pediatr Emerg Care. 1997;13(3):179-182.

4. Jha S. Stop hunting for zebras in Texas: end the diagnostic culture of "ruleout". BMJ. 2014;348:g2625.

5. Mold JW, Stein HF. The cascade effect in the clinical care of patients. N Engl J Med. 1986;314(8):512-514.

6. Shahrin L, Chisti MJ, Huq S, et al. Clinical Manifestations of Hyponatremia and Hypernatremia in Under-Five Diarrheal Children in a Diarrhea Hospital. J Trop Pediatr. 2016;62(3):206-212.

7. Wathen JE, MacKenzie T, Bothner JP. Usefulness of the serum electrolyte panel in the management of pediatric dehydration treated with intravenously administered fluids. Pediatrics. 2004;114(5):1227-1234

8. Practice parameter: the management of acute gastroenteritis in young children. American Academy of Pediatrics, Provisional Committee on Quality Improvement, Subcommittee on Acute Gastroenteritis. Pediatrics. 1996;97(3):424-435.

9. National Collaborating Centre for Women's and Children's Health. Diarrhoea and Vomiting Caused by Gastroenteritis: Diagnosis, Assessment and Management in Children Younger than 5 Years. London: RCOG Press; 2009.

10. Guarino A, Ashkenazi S, Gendrel D, et al. European Society for Pediatric Gastroenterology, Hepatology, and Nutrition/European Society for Pediatric Infectious Diseases evidence-based guidelines for the management of acute gastroenteritis in children in Europe: Update 2014. J Pediatr Gastroenterol Nutr. 2014;59(1):132-152.

11. Freedman SB, Gouin S, Bhatt M, et al. Prospective assessment of practice pattern variations in the treatment of pediatric gastroenteritis. Pediatrics. 2011;127(2):e287-e295.

12. Lind $\mathrm{CH}$, Hall M, Arnold DH, et al. Variation in Diagnostic Testing and Hospitalization Rates in Children With Acute Gastroenteritis. Hosp Pediatr. 2016;6(12):714-721.

13. Powell EC, Hampers LC. Physician variation in test ordering in the management of gastroenteritis in children. Arch Pediatr Adolesc Med. 2003;157(10):978-983.
- If initial testing is performed and indicates an expected value indicative of dehydration, do not repeat testing to demonstrate normalization as long as the child is clinically improving as expected.

\section{CONCLUSION}

Children presenting with mild-to-moderate dehydration should be treated with supportive measures in accordance with current guidelines. Electrolyte panels very rarely provide clinical information that cannot be garnered through a thorough history and PE. As in our clinical scenario, the laboratory values obtained may have led to potential harm, including overdiagnosis, painful procedures, and psychological distress. Without testing, the patient likely could have been appropriately treated with ORT and discharged from the ED.

Do you think this is a low-value practice? Is this truly a "Thing We Do for No Reason?" Share what you do in your practice and join in the conversation online by retweeting it on Twitter (\#TWDFNR) and liking it on Facebook. We invite you to propose ideas for other "Things We Do for No Reason" topics by emailing TWDFNR@hospitalmedicine.org.

Disclosure: The authors have nothing to disclose.

14. Steiner MJ, DeWalt DA, Byerley JS. Is this child dehydrated? JAMA. 2004;291(22):2746-2754

15. Sandhu BK, European Society of Pediatric Gastroenterology H, Nutrition Working Group on Acute D. Practical guidelines for the management of gastroenteritis in children. J Pediatr Gastroenterol Nutr. 2001;33(suppl 2):S36-S39.

16. Tieder JS, Robertson A, Garrison MM. Pediatric hospital adherence to the standard of care for acute gastroenteritis. Pediatrics. 2009;124(6):e1081-e1087.

17. Freedman SB, DeGroot JM, Parkin PC. Successful discharge of children with gastroenteritis requiring intravenous rehydration. J Emerg Med. 2014;46(1): 9-20.

18. Deyo RA. Cascade effects of medical technology. Annu Rev Public Health. 2002;23:23-44

19. Coon ER, Quinonez RA, Moyer VA, Schroeder AR. Overdiagnosis: how our compulsion for diagnosis may be harming children. Pediatrics. 2014;134(5):1013-1023

20. Florin TA, French B, Zorc JJ, Alpern ER, Shah SS. Variation in emergency department diagnostic testing and disposition outcomes in pneumonia. Pediatrics. 2013;132(2):237-244

21. Newman TB, Bernzweig JA, Takayama JI, Finch SA, Wasserman RC, Pantell $\mathrm{RH}$. Urine testing and urinary tract infections in febrile infants seen in office settings: the Pediatric Research in Office Settings' Febrile Infant Study. Arch Pediatr Adolesc Med. 2002;156(1):44-54.

22. McMurtry CM, Noel M, Chambers CT, McGrath PJ. Children's fear during procedural pain: preliminary investigation of the Children's Fear Scale. Health Psychol. 2011;30(6):780-788.

23. von Baeyer CL, Marche TA, Rocha EM, Salmon K. Children's memory for pain: overview and implications for practice. J Pain. 2004;5(5):241-249.

24. American Academy of Pediatrics. Section on Hospital Medicine. Rauch DA, Gershel JC. Caring for the hospitalized child: a handbook of inpatient pediatrics. Elk Grove Village, IL: American Academy of Pediatrics; 2013.

25. Bailey B, Gravel J, Goldman RD, Friedman JN, Parkin PC. External validation of the clinical dehydration scale for children with acute gastroenteritis. Acad Emerg Med. 2010;17(6):583-588

26. Friedman JN, Goldman RD, Srivastava R, Parkin PC. Development of a clinical dehydration scale for use in children between 1 and 36 months of age. $J$ Pediatr. 2004;145(2):201-207. 